\title{
Process Research On Cone-Shaped Micro-Nano Structures
}

\author{
Hsin-Chiang You ${ }^{1, *}$ and Yu-Hsien Lin ${ }^{2}$ \\ ${ }^{1}$ Department of Electronic Engineering, National Chin-Yi University of Technology, 41151 Taichung, Taiwan, R.O.C \\ ${ }^{2}$ Department of Electronic Engineering, National United University, 36003 Miaoli, Taiwan, R.O.C
}

Received: 2 Oct. 2013, Revised: 30 Dec. 2013, Accepted: 31 Dec. 2013

Published online: 1 Sep. 2014

\begin{abstract}
In this study, the manufacturing methods and imaging principles of cone-shaped arrays of micro-nano structure films were examined. Because optical films fabricated using cone-shaped arrays of micro-nano structures generate a double diffusion and produce diverse light effects on light sources, these optical films can be used effectively to enhance optical efficiency and can thus be used widely in liquid crystal displays. The experimental steps for producing cone-shaped arrays of micro-nano-structure films were as follows: First, a silicon wafer was spun off water, bake-dried, and applied with a photoresist coating, which was soft-baked on a hot plate. Next, the photoresist layer was exposed to light by using a stepper, and the wafer was then developed and hard-baked on a hot plate to produce cone-shaped arrays of micro-nano-structure films. This study proposes for the first time an exposure method applied on a wafer in which focus and exposure dosage of light are adjusted to produce films of cone-shaped micro-nano structures with distinct slopes. Using this method, films can be manufactured through micro-evolutionary changes in the array structure.
\end{abstract}

Keywords: Cone-shaped micro-nano structures, anti-reflective layer

\section{Introduction}

In many present-day optical components, the photoelectric effect is used to produce electrons and thereby light energy is converted into electrical energy. Thus, when fewer electro-optical components are used, less light energy is lost because of reflection and more light energy is absorbed, which results in light energy being used efficiently [1,2]. Therefore, applying an efficient anti-reflective layer during the production of optical components represents a critical technological step. When optical displays possess an anti-reflective layer on their surface $[3,4]$, the efficiency with which light energy is used is increased, and the distortion of images by reflected ambient light is decreased. Conventionally, to reduce the amount of ambient light that falls on a reflective substrate, multiple layers of the substrate are deposited, which lessens the light reflected on to the film that is produced. However, this fabrication process is both expensive and time-consuming. In this study, a new anti-reflective layer with a cone-shaped columnar micro-nano structure is proposed (Fig. 1).

Growing the proposed structure on the surface of optical components can make the components reflect less

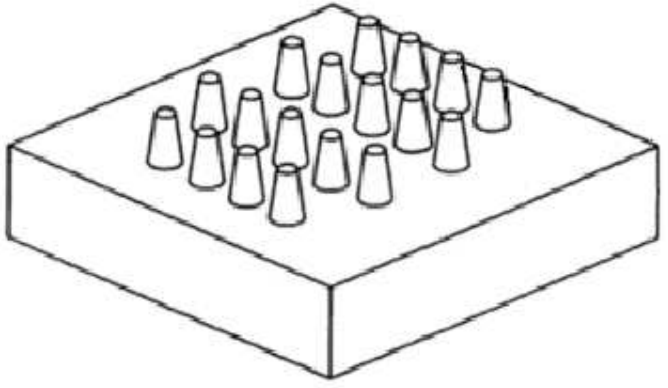

Fig. 1: The diagram of coned-shaped micro-nano columnar structure.

ambient light, and thus the optical components and displays can be used efficiently. The structure was developed by applying the principles of refraction, using cone-shaped micro-nano geometric shapes to increase the area of the surface that absorbs light energy. However, this micro-structure is unlike the anti-reflective materials

\footnotetext{
*Corresponding author e-mail: hcyou@ncut.edu.tw
} 


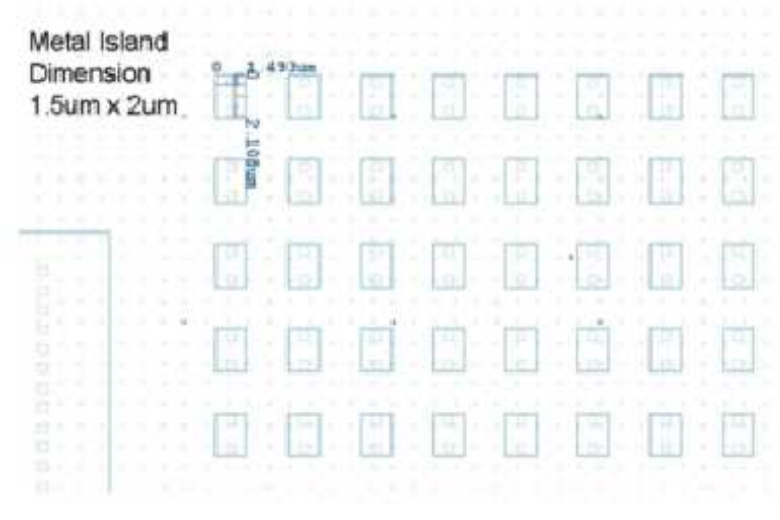

Fig. 2: The diagram of micro-nano structure arrays of masks.

used on substrates and has a distinct refractive index, which reduces the reflection of ambient light; thus, the proposed structure is strongly anti-reflective.

The cone-shape micro-nano structures were fabricated in this study by using lithography to produce abnormal structures. At a fixed thickness of the photoresist layer, focus depth and exposure dosages of light were changed to enable the post-exposure mask to transfer patterns on to the photoresist, which produced the size and shape changes required to obtain distinct micro-structure anomalies. Focusing the light on the topmost layer of the photoresist ensured that cone-shaped micro-nano structures were generated after the exposure.

\section{Experimental}

This report describes the manufacturing methods and imaging principles used for inducing evolutionary changes in arrays of cone-shaped columnar micro-nano structures. The changes in the cone-shaped micro-nano columnar structures were examined by 1) adjusting the focus depth from 0.5 to $1.9 \mu \mathrm{m}$, and 2) adjusting exposure dosages from 32 to $36 \mathrm{mj} / \mathrm{cm}^{2}$. The experimental steps were as follows: First, a silicon wafer was spun off water, bake-dried, and applied with a $3.5 \mu \mathrm{m}$ thick photoresist coating, which was soft-baked on a hot plate at $90^{\circ} \mathrm{C}$ for 1 min. Next, the wafer was placed in a stepper and the photoresist layer was exposed under an I-Line light source with a wavelength of $365 \mathrm{~nm}$; the mask used is shown in Fig. 2.

The wafer was baked after exposure at $120^{\circ} \mathrm{C}$ for $2 \mathrm{~min}$ and then developed immediately and hard-baked at $120^{\circ} \mathrm{C}$ for $2 \mathrm{~min}$ to generate the cone-shaped columnar micronano structures.

\section{Results and Discussion}

First, the effect adjusting focus depth on the changes in the cone-shaped columnar micro-nano structure is described. The experimental conditions are presented in Tables 1 and 2.

Table 1: The experimental conditions are: exposure to a fixed dose of $34 \mathrm{mj} / \mathrm{cm}^{2}$, and focus depth changing from $0.5 \mu \mathrm{m}$ to $1.1 \mu \mathrm{m}$. The top of crystal decreases from $1.124 \mu \mathrm{m}$ into $0.736 \mu \mathrm{m}$ and the bottom of crystal increases from $1.172 \mu \mathrm{m}$ into $1.484 \mu \mathrm{m}$.

\begin{tabular}{c|cccc}
\hline \hline $\mathrm{EF}=34 m j / \mathrm{cm}^{2}$ & \multicolumn{4}{c}{} \\
\hline Focus $(\mu \mathrm{m})$ & 0.5 & 0.7 & 0.9 & 1.1 \\
CD Top View $(\mu \mathrm{m})$ & 1.124 & 0.971 & 0.882 & 0.736 \\
CD Bottom View $(\mu m)$ & 1.172 & 1.378 & 1.423 & 1.484 \\
\hline \hline
\end{tabular}

Table 2: The experimental conditions are: exposure to a fixed dose of $34 \mathrm{mj} / \mathrm{cm}^{2}$, and focus depth changing from $1.3 \mu \mathrm{m}$ to $1.9 \mu \mathrm{m}$. The top of crystal decreases from $0.679 \mu \mathrm{m}$ into $0.103 \mu \mathrm{m}$ and the bottom of crystal increases from $1.531 \mu \mathrm{m}$ into $1.572 \mu \mathrm{m}$.

\begin{tabular}{c|cccc}
\hline \hline $\mathrm{EF}=34 \mathrm{mj} / \mathrm{cm}^{2}$ & & & & \\
\hline Focus $(\mu \mathrm{m})$ & 1.3 & 1.5 & 1.7 & 1.9 \\
CD Top View $(\mu \mathrm{m})$ & 0.679 & 0.503 & 0.248 & 0.103 \\
CD Bottom View $(\mu \mathrm{m})$ & 1.531 & 1.556 & 1.569 & 1.572 \\
\hline \hline
\end{tabular}

Figs. 3 and 4 show the cone-shaped columnar micronano structures obtained when the exposure dosage was fixed at $34.0 \mathrm{mj} / \mathrm{cm}^{2}$ and the focus depth was changed from 0.5 to $1.9 \mu \mathrm{m}$.

Fig. 3 presents scanning electron microscopy (SEM) images showing the crystal dimension from top and bottom views, and the figure also presents the tiled SEM views. Fig. 4 shows tiled SEM views of arrays of micro-nano structures. Fig. 3 shows the top crystal dimension. When the focus depth was changed from +0.5 to $+1.9 \mu \mathrm{m}$, the crystal dimension at the top decreased from 1.124 to $0.103 \mu \mathrm{m}$; this was mainly because the larger focus caused the top of the photoresist layer to absorb more energy, which reduced the crystal dimension. When the exposure was fixed at $34.0 \mathrm{mj} / \mathrm{cm}^{2}$ and the focus depth was changed from +0.5 to $+1.9 \mu m$, the crystal dimension at the top varied considerably. During the period when the focus depth is varied, the energy-absorption characteristics of the photoresist layer could change substantially: because the focus is positive, energy will be focused at the top layer of the photoresist and thereby cause the top layer of the photoresist to absorb a high proportion of the energy. The changes in crystal dimensions are reflected by the trends shown in Fig. 5 and in the histogram in Fig. 6.

When the focus depth was changed from +0.5 to $+1.9 \mu \mathrm{m}$, the crystal dimension at the bottom increased 

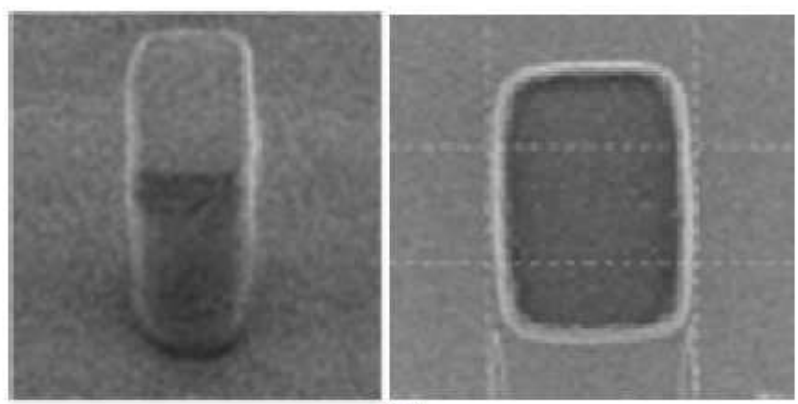

(a)

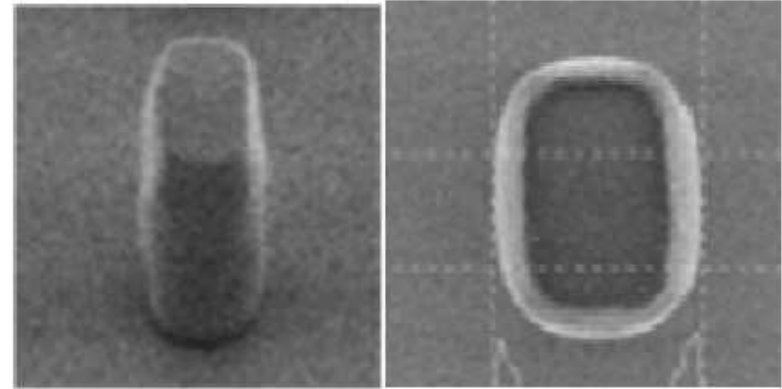

(b)
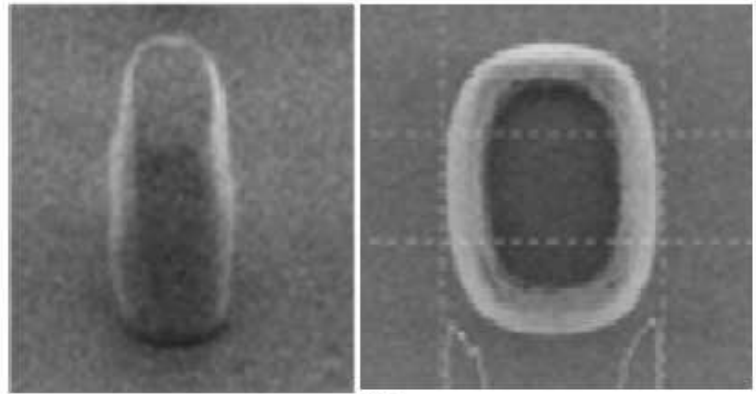

(c)

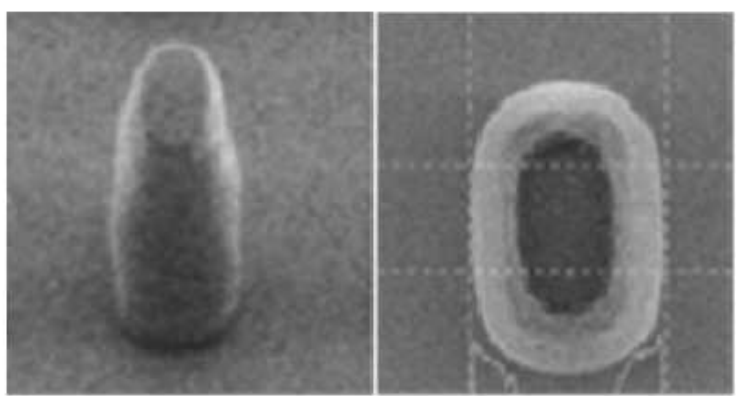

(d)

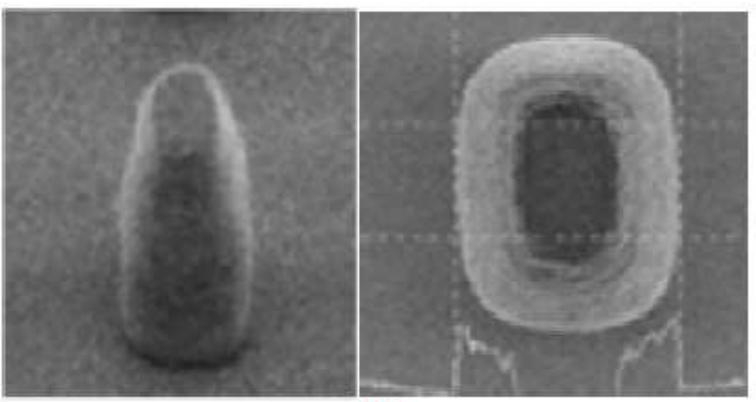

(e)

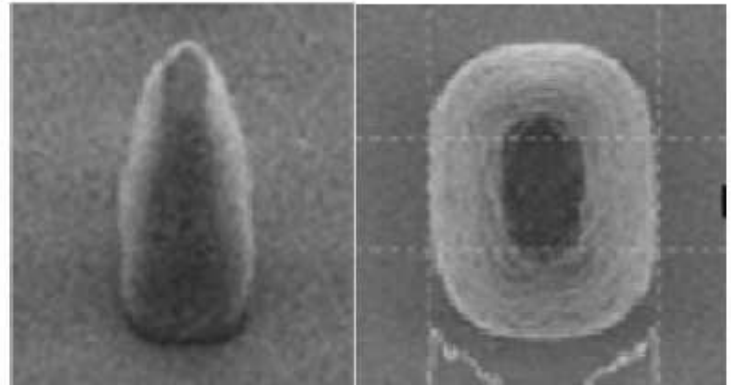

(f)

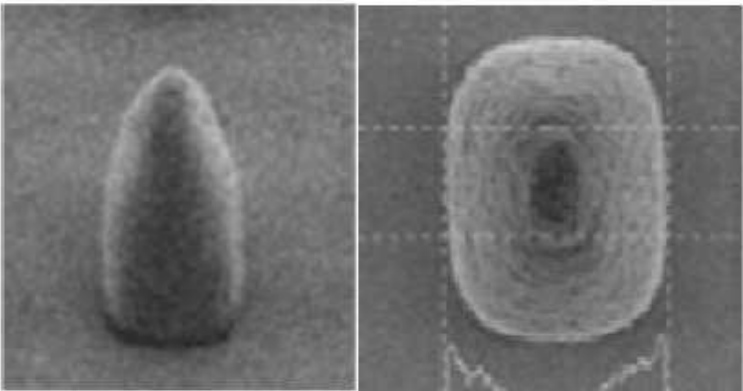

(g)

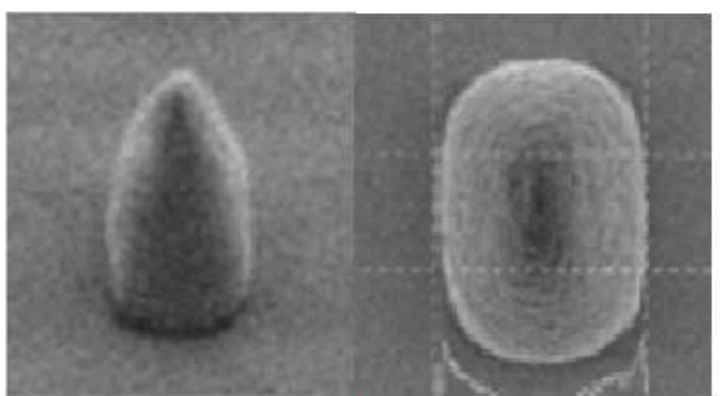

(h)

Fig. 3: The diagram of scanning electron microscope crystal dimension top and bottom views, and tile scanning electron microscope view. The experimental conditions are: exposure to a fixed dose of $34.0 \mathrm{mj} / \mathrm{cm}^{2}$, and focus depth changing from $0.5 \mu \mathrm{m}$ to $1.9 \mu \mathrm{m}$. (a) $0.5 \mu \mathrm{m}$ focus depth. (b) $0.7 \mu \mathrm{m}$ focus depth. (c) $0.9 \mu \mathrm{m}$ focus depth. (d) $1.1 \mu \mathrm{m}$ focus depth. (e) $1.3 \mu \mathrm{m}$ focus depth. (f) $1.5 \mu \mathrm{m}$ focus depth. (g) $1.7 \mu \mathrm{m}$ focus depth. (h) $1.9 \mu \mathrm{m}$ focus depth. 


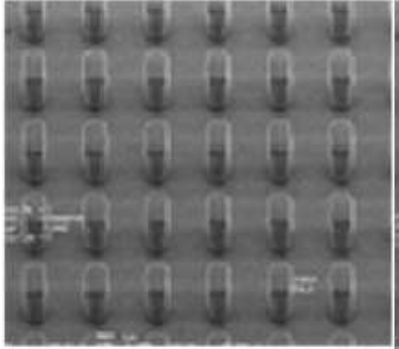

(a)

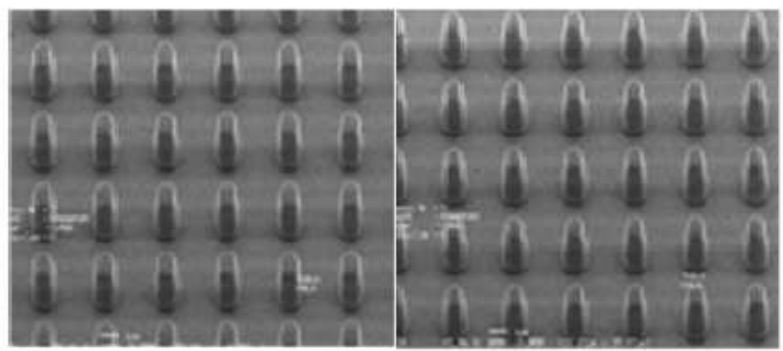

(c)

(d)

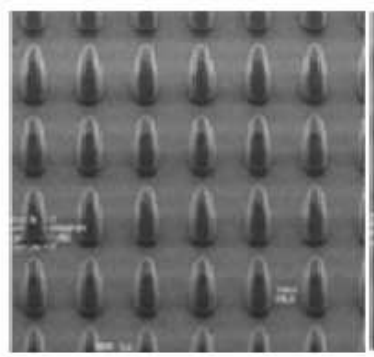

(e)
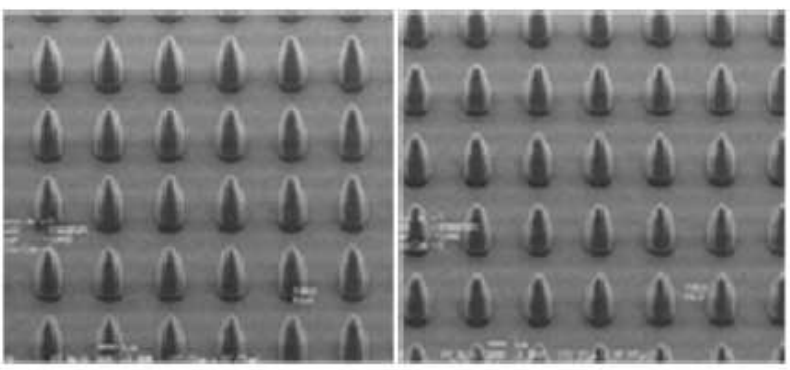

Fig. 4: The diagram of tile scanning electron microscope view of for the micro-nano structure arrays. The experimental conditions are: exposure to a fixed dose of $34.0 \mathrm{mj} / \mathrm{cm}^{2}$, and focus depth changing from $0.5 \mu \mathrm{m}$ to $1.9 \mu \mathrm{m}$. (a) $0.5 \mu \mathrm{m}$ focus depth. (b) $0.7 \mu \mathrm{m}$ focus depth. (c) $0.9 \mu \mathrm{m}$ focus depth. (d) $1.1 \mu \mathrm{m}$ focus depth. (e) $1.3 \mu \mathrm{m}$ focus depth. (f) $1.5 \mu \mathrm{m}$ focus depth. (g) $1.7 \mu \mathrm{m}$ focus depth. (h) $1.9 \mu \mathrm{m}$ focus depth.

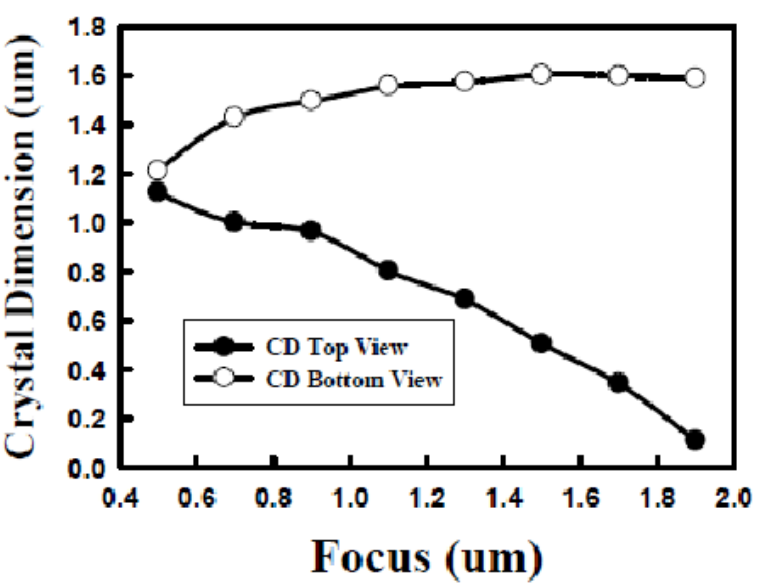

Fig. 5: The change trend charts for the top and bottom crystal dimension after exposure at a fixed dosage of $34.0 \mathrm{mj} / \mathrm{cm}^{2}$, and changing focus depths from $0.5 \mu \mathrm{m}$ to $1.9 \mu \mathrm{m}$.

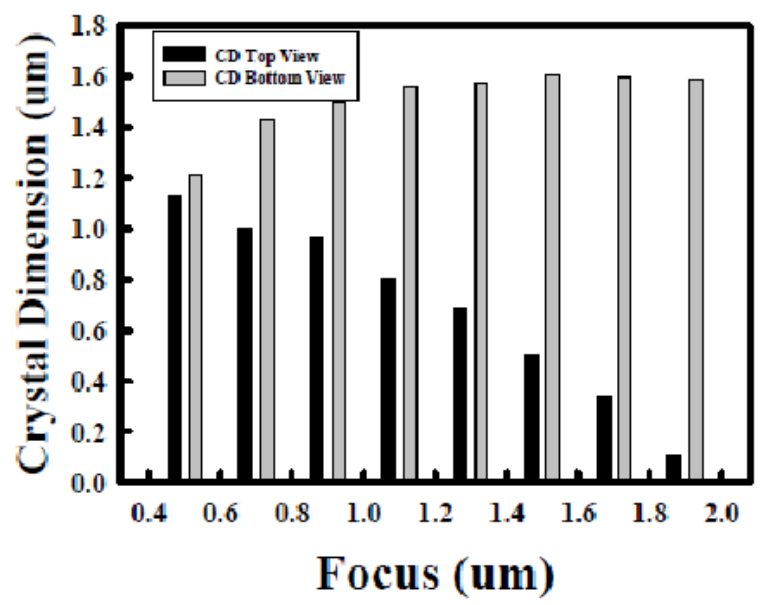

Fig. 6: The histogram change for the top and bottom crystal dimension after exposure at a fixed dosage of $34.0 \mathrm{mj} / \mathrm{cm}^{2}$, and changing focus depths from $0.5 \mu \mathrm{m}$ to $1.9 \mu \mathrm{m}$.

from 1.172 to $1.572 \mu \mathrm{m}$, mainly because the larger focus caused the bottom of the photoresist layer to absorb less energy, which resulted in an increase in the crystal dimension. When the exposure was fixed at $34.0 \mathrm{mj} / \mathrm{cm}^{2}$ and the focus depth was changed from +0.5 to $+1.1 \mu \mathrm{m}$, the crystal dimension at the bottom varied considerably and the energy-absorption characteristics of the photoresist changed substantially. However, when the focus depth was changed from +1.1 to $+1.9 \mu \mathrm{m}$, the crystal dimension at the bottom did not vary markedly. When changing focus depth, as the focus depth becomes larger, the top of the photoresist absorbs more energy and causes the bottom photoresist layer to absorb less energy, a trend that is shown in Fig. 5 and by the histogram in Fig. 6. Next, the effect of adjusting exposure energy on 
the change in the cone-shaped columnar micro-nano structures was examined. The experimental conditions are presented in Table 3.

Table 3: The experimental conditions are: the focus depth fixed at $+1.9 \mu \mathrm{m}$, and the exposure dosage changing from $32.0 \mathrm{mj} / \mathrm{cm}^{2}$ to $36.0 \mathrm{mj} / \mathrm{cm}^{2}$.

\begin{tabular}{c|ccc}
\hline \hline $\mathrm{EF}=19 \mathrm{mj} / \mathrm{cm}^{2}$ & & & \\
\hline Focus $\left(\mathrm{mj} / \mathrm{cm}^{2}\right)$ & 32 & 34 & 36 \\
CD Top View $(\mu \mathrm{m})$ & 0.111 & 0.102 & 0.088 \\
CD Bottom View $(\mu \mathrm{m})$ & 1.588 & 1.551 & 1.499 \\
\hline \hline
\end{tabular}

Figs. 7 and 8 show the cone-shaped columnar micronano structures obtained when the focus depth was fixed at $+1.90 \mu \mathrm{m}$ and the exposure dosages were changed from $32.0 \mathrm{mj} / \mathrm{cm}^{2}$ to $36.0 \mathrm{mj} / \mathrm{cm}^{2}$.

Fig. 7 presents SEM images showing top and bottom views of crystal dimensions and tiled SEM views. Fig. 8 shows a tiled SEM view of micro-nano-structure arrays. Fig. 7 shows that when exposure dosage was increased from 32.0 to $36.0 \mathrm{mj} / \mathrm{cm}^{2}$, the crystal dimension decreased from 0.111 to $0.088 \mu \mathrm{m}$; this was mainly because the larger exposure dosage caused the photoresist layer to absorb more energy, which resulted in the crystal dimension becoming smaller. When the focus depth was fixed at $+1.90 \mu \mathrm{m}$ and the exposure dosage was increased from $32.0 \mathrm{mj} / \mathrm{cm}^{2}$ to $36.0 \mathrm{mj} / \mathrm{cm}^{2}$, the change in the crystal dimension at the top became smaller and the trend appeared flat, which was because the photoresist layer had reached its absorption limit under these conditions. The trends are shown in Fig. 9 and in the histogram in Fig. 10.

Figs. 9 and 10 show that when the exposure dosage was increased from 32.0 to $36.0 \mathrm{mj} / \mathrm{cm}^{2}$, crystal dimensions at the bottom decreased from 1.588 to $1.499 \mu \mathrm{m}$; this was mainly because the larger exposure dosage caused the photoresist layer to absorb more energy, which resulted in the crystal dimension becoming smaller. When the focus depth was fixed at $+1.90 \mu \mathrm{m}$ and the exposure dosage was increased from 32.0 to $36.0 \mathrm{mj} / \mathrm{cm}^{2}$, the crystal dimension at the top became $21 \%$ smaller, whereas the crystal dimension at the bottom became only $6 \%$ smaller. Thus, using greater exposure energy, a cone-shaped columnar micro-nano structure with a larger slope gradient can be fabricated.

\section{Conclusions}

The experiments in this study showed that when positive focus was maintained and the light intensity was focused on the top layer of the photoresist, cone-shaped columnar micro-nano structures were created. The experimental results demonstrated that with the focus depth at $1.9 \mu \mathrm{m}$ and exposure dosage at $34.0 \mathrm{mj} / \mathrm{cm}^{2}$, micro-nano

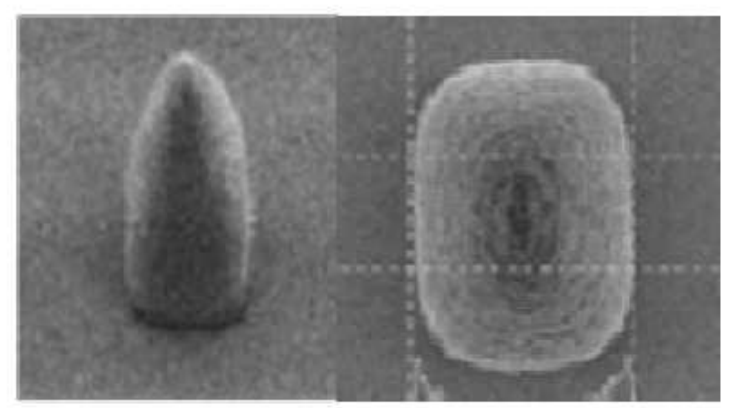

(a)

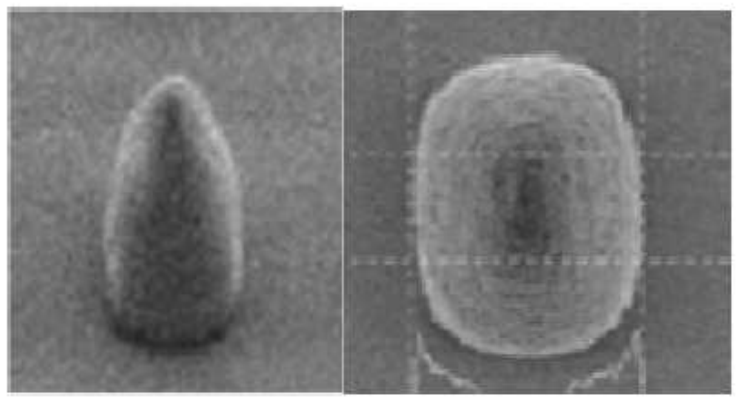

(b)

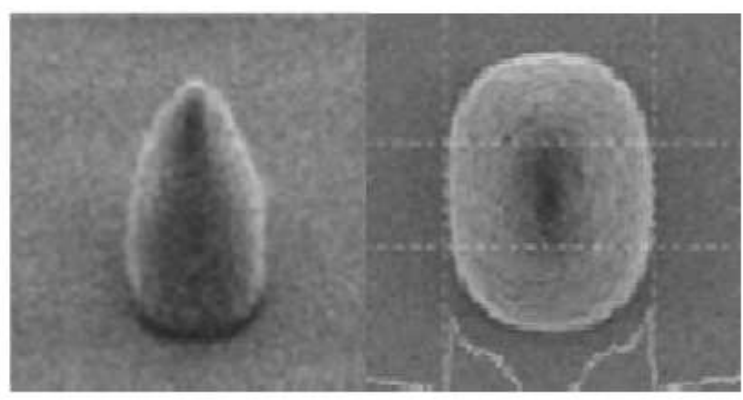

(c)

Fig. 7: The diagram of scanning electron microscope crystal dimension top and bottom views, and tile scanning electron microscope view. The experimental conditions are: the focus depth fixed at $+1.9 \mu \mathrm{m}$, and the exposure dosage changing from $32.0 \mathrm{mj} / \mathrm{cm}^{2}$ to $36.0 \mathrm{mj} / \mathrm{cm}^{2}$. (a) $32.0 \mathrm{mj} / \mathrm{cm}^{2}$. (b) $34.0 \mathrm{mj} / \mathrm{cm}^{2}$. (c) $36.0 \mathrm{mj} / \mathrm{cm}^{2}$.

structures can be obtained with crystal dimensions of $0.103 \mu \mathrm{m}$ at the top and $1.572 \mu \mathrm{m}$ at the bottom. In this study, the minimal dimension obtained at the top of the cone-shaped columnar micro-nano structures was $0.103 \mu \mathrm{m}$. When focus was varied, the line width of the micro-structures at the top was not decreased readily. Furthermore, when the focus depth was fixed and the light intensity was focused on the top of the photoresist layer, the exposure increased, and both the top and bottom crystal dimensions became smaller because the increase 


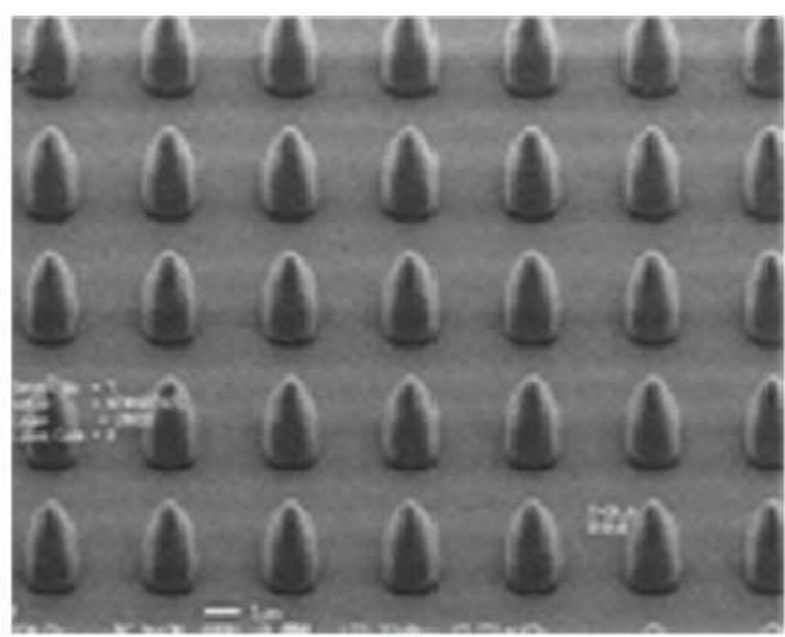

(a)

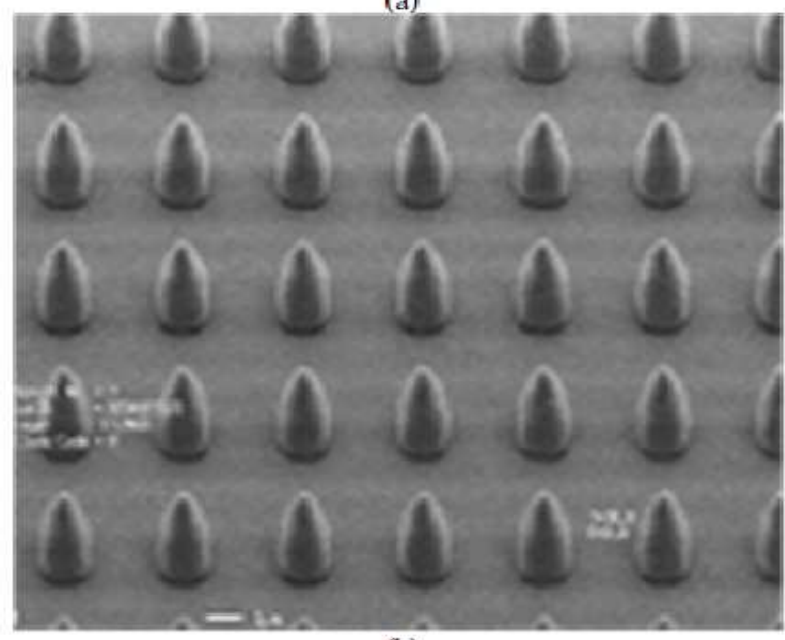

(b)

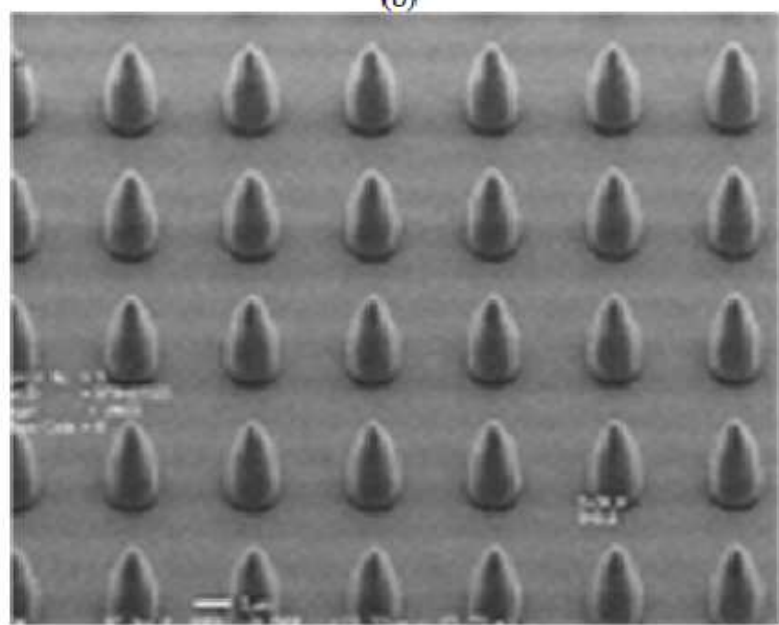

(c)

Fig. 8: The tile scanning electron microscope view of the micronano structure arrays. The experimental conditions are: the focus depth fixed at $+1.9 \mu \mathrm{m}$, and the exposure dosage changing from $32.0 \mathrm{mj} / \mathrm{cm}^{2}$ to $36.0 \mathrm{mj} / \mathrm{cm}^{2}$. (a) $32.0 \mathrm{mj} / \mathrm{cm}^{2}$. (b) $34.0 \mathrm{mj} / \mathrm{cm}^{2}$. (c) $36.0 \mathrm{mj} / \mathrm{cm}^{2}$.

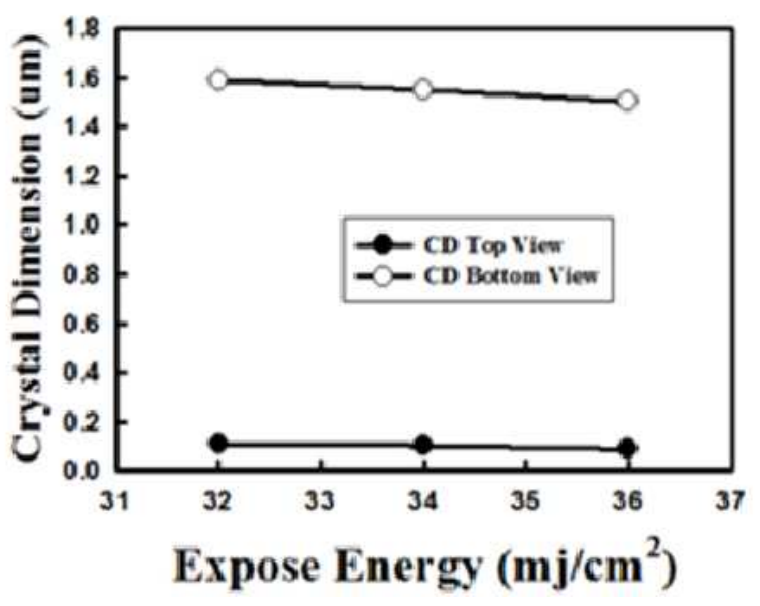

Fig. 9: The change trend chart for the top and bottom crystal dimension after the focus fixed at $1.9 \mu \mathrm{m}$, and exposure dosage changing from $32.0 \mathrm{mj} / \mathrm{cm}^{2}$ to $36.0 \mathrm{mj} / \mathrm{cm}^{2}$.

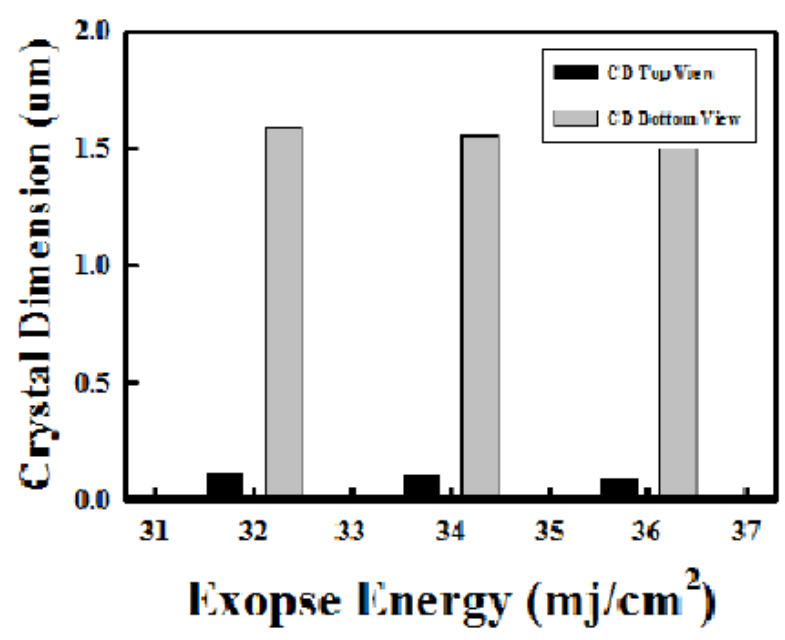

Fig. 10: The histogram change for the top and bottom crystal dimension after the focus fixed at $1.9 \mu \mathrm{m}$, and exposure dosage changing from $32.0 \mathrm{mj} / \mathrm{cm}^{2}$ to $36.0 \mathrm{mj} / \mathrm{cm}^{2}$.

in exposure energy caused the photoresist to absorb more energy. However, when the light intensity was focused on the top of the photoresist layer, the top crystal dimension became $21 \%$ smaller, whereas the bottom crystal dimension became only $6 \%$ smaller. Thus, by using greater exposure energy, cone-shaped columnar micro-nano structures can be created with considerable disparity in crystal dimensions: $0.088 \mu \mathrm{m}$ at the top and $1.499 \mu \mathrm{m}$ at the bottom. 


\section{Acknowledgement}

This work was supported by the National Science Council, Taiwan, under Contract Nos. NSC 1022221 E 167 - 033.

\section{References}

[1] Z. Yu, H. Gao, W. Wu, H. Ge, and S. Y. Chou, Fabrication of large area subwavelength antireflection structures on Si using trilayer resist nanoimprint lithography and liftoff, J. Vac. Sci. Technol., 2874-2877 (2003).

[2] H. L. Chen, K. T. Huang, C. H. Lin, W. Y. Wang, and W. Fan, Fabrication of sub-wavelength antireflective structures in solar cellsby utilizing modified illumination and defocus techniques inoptical lithography, Microelectronic Engineering, 84, 750-754 (2007).

[3] Y. H. Kang, S. S. Oh, Y. S. Kim, and C. G. Choi, Fabrication of antireflection nanostructures by hybrid nanopatterning lithography, Microelectronic Engineering, 87, 125-128 (2010).

[4] M. Cao, X. Song, J. Zhai, J. Wang, and Y. Wang, Fabrication of Highly Antireflective Silicon Surfaces with Superhydrophobicity, J. Phys. Chem. B, 110, 13072-13075 (2006).

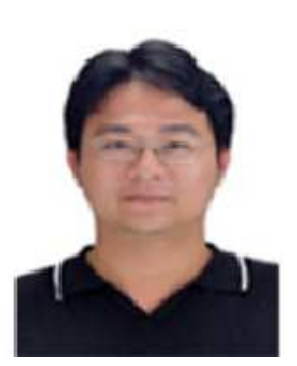

Hsin-Chiang You was born in Changhua, Taiwan, R.O.C., on May 23, 1977. $\mathrm{He}$ received the B.S.and M.S. degrees in electrical engineering from Feng Chia University, Taichung, Taiwan, in 1999 and 2001, respectively. He received the Ph.D. degree in electronics engineering from National Chiao-Tung University, Hsinchu, Taiwan, R.O.C., in 2006. His Ph.D. dissertation research focused on nano-devices and memories. From 2007 to 2009, he was with the Department of Computer Science and Information Engineering, Asia University, Taichung, Taiwan, as an Assistant Professor. In 2009, he joined the Department of Electronic Engineering, National Chin-Yi University of Technology, Taichung, Taiwan. R.O.C., and currently he is an Assistant Professor. His research interests include nano-devices and flexible devices.

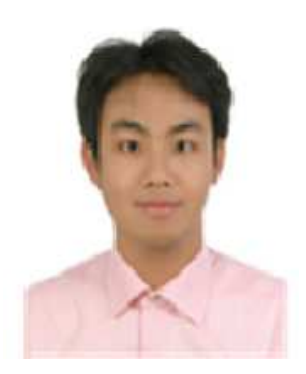

Yu-Hsien Lin was born in Yi-Lan, Taiwan, R.O.C., on June 18, 1979. $\mathrm{He}$ received the B.S., M.S. and Ph.D. degrees in electronics engineering from National Chiao-Tung University, Hsinchu, Taiwan, R.O.C., in 2001, 2002, and 2006 respectively. His Ph.D.

dissertation research focused on engineering and physics of advanced memory devices (in particular, nanocrystal based). From 2006 to 2010, he was with the Taiwan Semiconductor Manufacturing Company (TSMC), Taiwan, as a Principle Engineer working on the research and design for N40/N20 process integration and device performance improvement. In 2011, he joined the Department of Electronic Engineering, National United University, Miaoli, Taiwan. R.O.C., and currently he is an Assistant Professor. His research interests include novel nonvolatile memory devices, high-k dielectric materials for CMOS devices, and poly-Si thin film transistors. 